\title{
ARTERIAL PRESSURE, PHYSIQUE, AND OCCUPATION
}

\author{
BY \\ C. R. LOWE \\ Department of Social and Occupational Medicine, Welsh National School of Medicine, and Department of Social \\ Medicine, University of Birmingham
}

What influence occupation has upon arterial pressure is uncertain. Ischaemic heart disease is known to be more common among men in sedentary than in heavy occupations (Morris, Heady, Raffle, Roberts, and Parks, 1953; Brown, Davidson, McKeown, and Whitfield, 1957; Morris and Crawford, 1958) and there is good evidence that the attack rate increases with increasing arterial pressure (Brown and others, 1957). One would expect to find, therefore, that men in sedentary occupations tend to have higher pressures than men doing heavy work. The results of a survey carried out by Miall and Oldham (1958) in South Wales appear to be in line with this expectation-men doing light work were reported to have had somewhat higher pressures than those doing heavy work. But a study by Edwards, McKeown, and Whitfield (1959) in Birmingham produced results which suggested the opposite of this. Arterial pressure was measured in 1,723 representative men over the age of 60 and was found to be a little higher in Social Classes IV and V (semi-skilled and unskilled occupations) than in Classes I and II (professional and intermediate occupations).

No explanation has been offered for the difference between the findings in South Wales and Birmingham, and national data on occupational mortality do little to clarify the position (Registrar General, 1954). For the years 1949-53, mortality attributed to to hypertension among men aged 20-64 appeared to follow the same pattern as mortality attributed to coronary disease, and tended to decrease, although not so steeply, from Social Class I to V. On the face of it this appears to support the findings in South Wales. The Registrar General warns, however, that trends in the social class mortality of men aged 20-64 must be interpreted with caution, for when the statements of occupation on 10,000 death certificates issued 3 weeks after the census were compared with the statements in the related census schedules, a tendency to upgrade social class on the death certificate was disclosed which would have been sufficient to increase the apparent mortality for Classes I and II by perhaps as much as 25 per cent. So the social class trend in mortality from hypertension can probably be discounted.

The relation between arterial pressure (or, more correctly, sphygmomanometer reading) and weight and height appears, at first sight, to be more straightforward. Two recent and very large surveys have confirmed what clinical impression and many smaller surveys have suggested - that blood pressure readings increase fairly consistently with weight and are little if at all related to height. In the first of these, reported by Bøe, Humerfelt, and Wedervang (1957), blood pressure, height, and weight were recorded for 68,000 adults in Bergen at the time of a compulsory mass $x$-ray campaign. The second consisted of a stratified sample (235,000 males and 45,000 females) of ordinary insurance issued by 26 companies in the United States and Canada between 1935 and 1953 (Society of Actuaries, 1959). In both populations blood pressure showed a moderate increase with weight at constant age and height and, if anything, a slight decrease with height at constant age and weight.

This apparent relation between arterial pressure and body build is complicated by the influence of arm circumference, which itself is highly correlated with weight. Ragan and Bordley (1941) compared direct arterial pressure readings on one arm with simultaneous auscultatory sphygmomanometer readings on the other for 51 subjects and found that sphygmomanometer readings tended to be too low for small arms and too high for large ones. The number of observations they made was admittedly small and the pattern uncovered was not very consistent; nevertheless, their findings suggest that some (possibly all) of the observed association 
between arterial pressure and weight may in fact be due to the effect of arm circumference upon sphygmomanometer reading. Since physique and occupation are likely to be related to one another, it seems possible that the confusion in the literature about the association between arterial pressure and physical demands of occupation may have arisen for the same reason. In what follows, these difficulties are explored in relation to a survey of arterial pressure among the employees of a large electrical engineering firm in the Midlands.

\section{Method}

The data presented relate to 5,239 men and 1,572 women employed by the General Electric Company in Birmingham (Table 1). These men and women constituted 72 per cent. of the works' population, but they were not in the strict sense of the word a random sample. They were employees who accepted the offer of a chest $x$ ray when a mobile mass radiography unit visited the firm in March, 1960. However, all occupations were included-from works manager to serviceman-and, for the purposes of this communication, the sample can be accepted as reasonably representative of persons employed in industry in the Midlands.

TABLE I

THE SAMPLE

\begin{tabular}{|c|c|c|c|c|c|c|c|c|}
\hline \multirow{2}{*}{\multicolumn{2}{|c|}{ Sex }} & \multicolumn{6}{|c|}{ Age Group (yrs) } & \multirow{2}{*}{ Total } \\
\hline & & $15-19$ & $20-29$ & $30-39$ & $40-49$ & $50-59$ & $60-69$ & \\
\hline $\begin{array}{l}\text { Male } \\
\text { Female }\end{array}$ & . & $\begin{array}{l}459 \\
375\end{array}$ & $\begin{array}{r}1,260 \\
428\end{array}$ & $\begin{array}{r}1,244 \\
248\end{array}$ & $\begin{array}{r}1,056 \\
293\end{array}$ & $\begin{array}{l}887 \\
189\end{array}$ & $\begin{array}{r}333 \\
39\end{array}$ & $\begin{array}{l}5,239 \\
1,572\end{array}$ \\
\hline Total & . & 834 & 1,688 & 1,492 & 1,349 & 1,076 & 372 & 6,811 \\
\hline
\end{tabular}

As the employees attended for $x$ ray examination, their blood pressures were recorded and they were weighed and measured. To measure arterial pressure twelve doctors were employed on a sessional basis. Four were present at each half-day session but, apart from ruling that no doctor should work for two consecutive sessions, no attempt was made to randomize their attendance, and the number of employees seen by each of the doctors over the 3 weeks of the survey varied considerably (range 217-712). Four new mercury sphygmomanometers with standard cuffs were used; systolic pressure was read as the point at which sounds first appeared, and diastolic pressure as the point at which they became muffled. When mercury manometer measurements are read to the nearest 2 or $5 \mathrm{~mm}$. $\mathrm{Hg}$, almost all observers show a conspicuous preference for numbers ending in 0 . Miall and Oldham (1958) showed that, by recording to the 5 or 0 below the point at which sounds appear or change, this tendency can be substantially reduced, so this was the method adopted in the present survey (e.g. readings from 120 to, but not including, 125 were recorded as 120 , and from 125 to, but not including, 130 as 125); mean values calculated from the data have accordingly been adjusted by adding $2.5 \mathrm{~mm}$.

Employees were asked to remove their shoes, overalls, coats, and waistcoats before they were measured. Weights were recorded to the nearest pound and were measured on a large spring balance which was checked against a known weight at the end of each session. Height was measured against a standard wall scale and recorded to the half inch below the observed point. Arm circumference was measured on a flexible steel tape at the mid-point of the right arm, with the arm hanging loosely by the side, and was recorded to the nearest half centimetre below the observed measurement.

In addition, information was collected about age, marital state, number of children, smoking habits, works status (monthly staff, weekly staff or works), and age of parents (present age for a living parent and age at death for a dead parent). Works check number was also recorded and provided a key to occupation and location within the factory. With its help it was possible to define precisely each person's job and to categorize it broadly according to the physical and mental demands it made. The demands were grouped as sedentary, light, or moderate to heavy (physically) and as minimum, moderate, or severe (intellectually).

Arterial Pressure in Relation to Age and Sex

There is now abundant evidence that, in the general population, arterial pressure has a continuous and unimodal distribution which flattens and becomes increasingly more skew to the right with age (Hamilton, Pickering, Roberts, and Sowry, 1954a; Bøe and others, 1957). In a previous publication, Lowe and McKeown (1962) have shown that the systolic and diastolic pressures recorded for the population of employed males in the present survey conform to this general pattern. The Birmingham data are also in agreement with material from other large-scale surveys in disclosing a sex difference in the relation between systolic pressure and age. In early adult life, females had lower mean systolic, and to a lesser extent diastolic, pressures than males; in middle life there was little difference between the sexes; in later life females had distinctly higher pressures than males (Table II and Fig. 1 opposite).

Clearly, blood pressure measurements from one population cannot be compared with measurements 
TABLE II

ARTERIAL PRESSURE RELATED TO SEX AND AGE*

\begin{tabular}{|c|c|c|c|c|c|c|c|c|}
\hline \multirow{3}{*}{$\begin{array}{c}\text { Age Group } \\
\text { (yrs) }\end{array}$} & \multicolumn{4}{|c|}{ Males } & \multicolumn{4}{|c|}{ Females } \\
\hline & \multicolumn{2}{|c|}{ Systolic Pressure } & \multicolumn{2}{|c|}{ Diastolic Pressure } & \multicolumn{2}{|c|}{ Systolic Pressure } & \multicolumn{2}{|c|}{ Diastolic Pressure } \\
\hline & Mean & S.D. & Mean & S.D. & Mean & S.D. & Mean & S.D. \\
\hline $\begin{array}{l}15-19 \\
20-29 \\
30-39 \\
40-49 \\
50-59 \\
60-69\end{array}$ & $\begin{array}{l}128 \cdot 5 \\
126 \cdot 7 \\
128 \cdot 5 \\
131 \cdot 8 \\
139 \cdot 3 \\
148 \cdot 6\end{array}$ & $\begin{array}{l}17 \cdot 2 \\
15 \cdot 9 \\
17 \cdot 1 \\
19 \cdot 1 \\
23 \cdot 1 \\
26 \cdot 1\end{array}$ & $\begin{array}{l}72 \cdot 5 \\
75 \cdot 7 \\
79 \cdot 6 \\
82 \cdot 2 \\
85 \cdot 0 \\
88 \cdot 0\end{array}$ & $\begin{array}{l}11.0 \\
10.6 \\
11.5 \\
11.9 \\
13.6 \\
14.4\end{array}$ & $\begin{array}{l}119.8 \\
123.4 \\
126.8 \\
134.9 \\
139.7 \\
155.0\end{array}$ & $\begin{array}{l}14 \cdot 4 \\
14 \cdot 2 \\
18 \cdot 4 \\
22 \cdot 2 \\
22 \cdot 4 \\
28 \cdot 3\end{array}$ & $\begin{array}{l}71 \cdot 4 \\
74 \cdot 9 \\
78 \cdot 1 \\
83 \cdot 4 \\
84 \cdot 0 \\
91 \cdot 2\end{array}$ & $\begin{array}{r}9.7 \\
10.5 \\
12.0 \\
12.8 \\
12.8 \\
15.9\end{array}$ \\
\hline
\end{tabular}

- The number of persons in each age group is the same as for the corresponding age group in Table I.

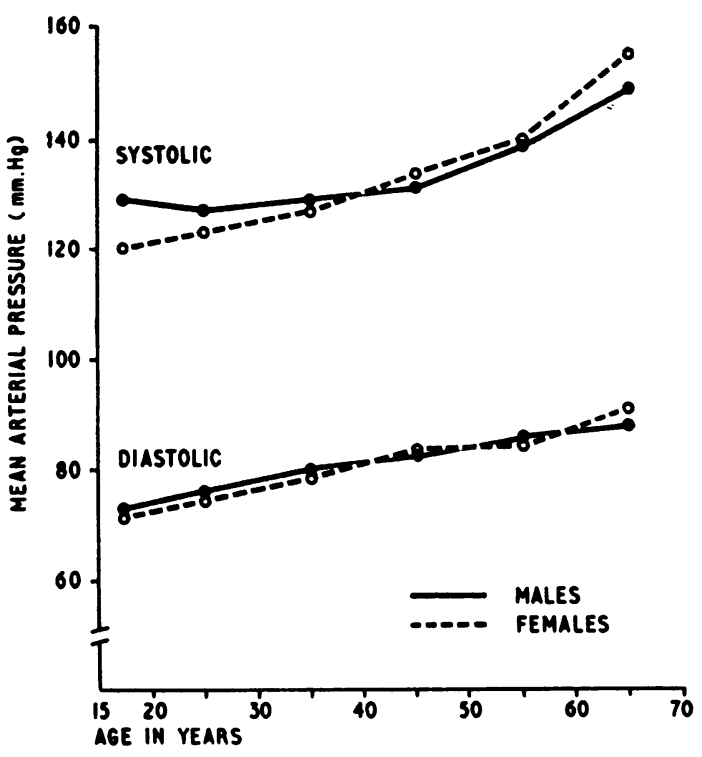

Fig.1.-Mean arterial pressure related to age and sex.

from another without correcting for age and sex. This difficulty is well recognized, and for small samples with a wide age range is probably insuperable. Fortunately, the number of men included in the present survey was large enough to permit an age-specific examination of pressure, physique, and occupation without recourse to age standardization or to the age-adjusted summarizing index devised by Hamilton and others (1954b). In what follows, therefore, only data relating to males are analysed and a decennial age grouping has been used because it provides reasonably adequate numbers in the appropriate weight, height, arm circumference, and occupational sub-groups. There was, of course, some change in mean pressure with age within each 10-year grouping, but for the present purpose this has been ignored.

\section{Observer VARIATION}

The mercury sphygmomanometer seems almost designed to provide maximum scope for observer variation. To read it the observer must relate a change in the quality and intensity of a rhythmic thud to the position of a falling and oscillating miniscus of mercury on a scale calibrated at 2-mm. intervals but numbered with the even "10s" on one side and the odd " 10 " on the other. The subjective element is so pronounced that, as Lowe and McKeown (1962) have shown, observers appear to establish their own norms, some reading consistently high and others consistently low. Table III indicates the range of observer variation in the reading of systolic pressure in the present survey. Observer A examined 195 males in the age group 20-39 and recorded a mean systolic pressure of $120 \cdot 0 \pm 0 \cdot 9$. Observer $L$ examined 164 males in the same age group and recorded a mean pressure of $135 \cdot 4 \pm 1 \cdot 4$, a difference of $15 \cdot 4 \pm 1 \cdot 7 \mathrm{~mm}$. The Table also provides a measure of the consistency with which each observer read high or low. The rank correlation coefficient for the twelve mean readings of systolic pressure for males aged 20-39 and males aged 40-59 was $+0 \cdot 87$.

TABLE III

VARIATION IN MEAN SYSTOLIC PRESSURE (mm. Hg) OF MALES AGED 20-39 AND 40-59 AS READ BY TWELVE OBSERVERS

\begin{tabular}{c|c|c|c|c}
\hline \multirow{2}{*}{ Observer } & \multicolumn{2}{|c|}{ Males aged 20-39 } & \multicolumn{2}{c}{ Males aged 40-59 } \\
\cline { 2 - 5 } & $\begin{array}{c}\text { Systolic Pressure } \\
\text { Mean and S.E. }\end{array}$ & $\begin{array}{c}\text { Rank } \\
\text { Order* }\end{array}$ & $\begin{array}{c}\text { Systolic Pressure } \\
\text { Mean and S.E. }\end{array}$ & $\begin{array}{c}\text { Rank } \\
\text { Order* }\end{array}$ \\
\hline A & $120.0+0.9$ & 1 & $125.2 \pm 1.2$ & 1 \\
B & $121.7 \pm 1.5$ & 2 & $126.8 \pm 2.4$ & 2 \\
C & $122.4 \pm 1.3$ & 3 & $136.6 \pm 2.3$ & 6 \\
D & $124.8 \pm 1.4$ & 4 & $130.3 \pm 2.0$ & 3 \\
E & $125.2 \pm 0.8$ & 5 & $132.6 \pm 1.1$ & 4 \\
F & $126.8 \pm 1.0$ & 6 & $136.8 \pm 1.3$ & 8 \\
G & $127.8 \pm 0.9$ & 7 & $136.7 \pm 1.5$ & 7 \\
H & $129.5 \pm 1.6$ & 8 & $139.3 \pm 2.4$ & 10 \\
I & $130.0 \pm 1.0$ & 9 & $135.0 \pm 1.5$ & 5 \\
J & $131.3 \pm 1.3$ & 10 & $137.6 \pm 1.6$ & 9 \\
K & $132.2 \pm 0.9$ & 11 & $141.2 \pm 1.5$ & 12 \\
L & $135.4 \pm 1.4$ & 12 & $140.2 \pm 2.0$ & 11 \\
\hline
\end{tabular}

* Spearman's rank correlation coefficient $\varrho=+0 \cdot 87$ 
Table IV gives the mean and standard error of diastolic pressure as read by the twelve observers for males in the same two age groups (20-39 and 40-59). Once again, there are very considerable and consistent differences between readings by the different observers.

TABLE IV

VARIATION IN MEAN DIASTOLIC PRESSURE (mm, $\mathrm{Hg}$ ) OF MALES AGED 20-39 AND 40-59 AS READ BY TWELVE OBSERVERS

\begin{tabular}{|c|c|c|c|c|}
\hline \multirow{2}{*}{ Observer } & \multicolumn{2}{|c|}{ Males aged 20-39 } & \multicolumn{2}{|c|}{ Males aged $40-59$} \\
\hline & $\begin{array}{l}\text { Diastolic Pressure } \\
\text { Mean and S.E. }\end{array}$ & $\begin{array}{c}\text { Rank } \\
\text { Order* }\end{array}$ & $\begin{array}{l}\text { Diastolic Pressure } \\
\text { Mean and S.E. }\end{array}$ & $\underset{\text { Order* }}{\text { Rank }}$ \\
\hline $\begin{array}{l}\mathbf{G} \\
\mathbf{K} \\
\mathbf{C} \\
\mathbf{B} \\
\mathbf{F} \\
\mathbf{I} \\
\mathbf{J} \\
\mathbf{A} \\
\mathbf{D} \\
\mathbf{L} \\
\mathbf{E} \\
\mathbf{H}\end{array}$ & $\begin{array}{l}68.0 \pm 0.5 \\
68.6 \pm 0.6 \\
68.9 \pm 0.8 \\
69.3 \pm 1.0 \\
71.4 \pm 0.7 \\
72.1 \pm 0.7 \\
72.4 \pm 0.8 \\
72.4 \pm 0.7 \\
74.6 \pm 0.8 \\
78.8 \pm 0.9 \\
79.4 \pm 0.6 \\
81.5 \pm 1.3\end{array}$ & $\begin{array}{l}1 \\
2 \\
3 \\
4 \\
5 \\
6 \\
7 \cdot 5 \\
7 \cdot 5 \\
9 \\
10 \\
11 \\
12\end{array}$ & $\begin{array}{l}75.6 \pm 0.8 \\
76.5 \pm 0.9 \\
76.6 \pm 1.3 \\
74.9 \pm 1.7 \\
77.7 \pm 0.8 \\
75.6 \pm 0.8 \\
76.8 \pm 0.9 \\
78.3 \pm 0.8 \\
78.9 \pm 0.9 \\
83.0 \pm 1.3 \\
84.2 \pm 0.7 \\
90.7 \pm 1.7\end{array}$ & $\begin{array}{l}2 \cdot 5 \\
4 \\
5 \\
1 \\
7 \\
2 \cdot 5 \\
6 \\
8 \\
9 \\
10 \\
11 \\
12\end{array}$ \\
\hline
\end{tabular}

* Spearman's rank correlation coefficient

Comparisons have often been made between the mean level of pressure recorded in one population in relation to age and other variables, with that recorded by a different observer or observers in another population. Evidently this is of doubtful value, for any difference uncovered is more likely to be due to observer variation than to a difference in the mean level of arterial pressure in the two populations examined. It also seems that internal comparisons made with data from the pooled observations of several observers are open to suspicion unless standardized for observer distribution at every stage of the comparison. However, Table $\mathrm{V}$ suggests a way in which this lengthy procedure may be avoided. It indicates that, although each of the twelve observers was consistent in his own reading of systolic and diastolic pressure, the level at which he read the former gave no information about the level at which he might be expected to read the latter. The rank order correlation coefficient for the mean readings of the twelve observers for systolic pressure and for diastolic pressure was +.089 in men aged $20-39$ and +.072 in men aged 40-59. It seems reasonable to assume therefore that, if in what follows a trend in relation to any variable (e.g. weight or physical demands of occupation) is demonstrated for both systolic and diastolic pressure, it is unlikely to be due to observer variation.

\section{Influence of PhysiQue}

Table VI (opposite) and Fig. 2 summarize the relation between arterial pressure and weight.

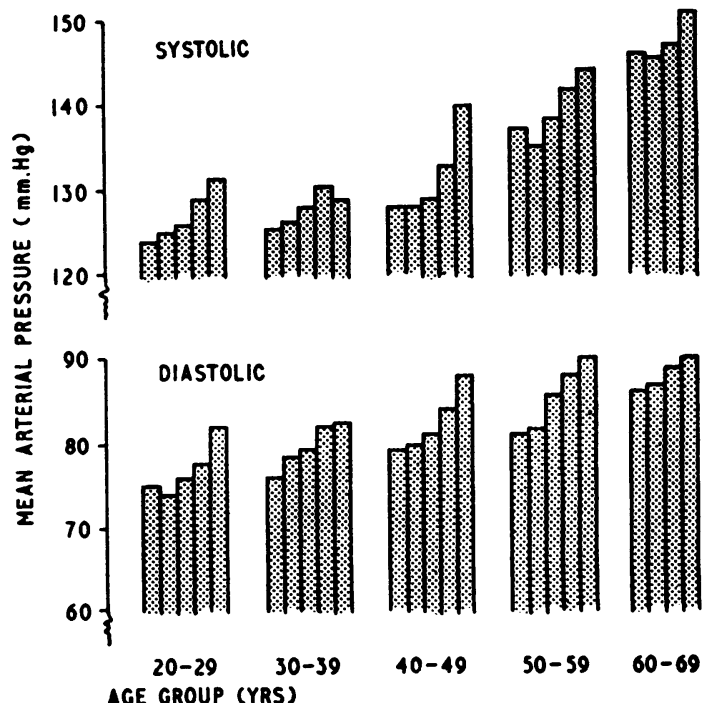

FIG. 2.--Mean arterial pressure related to weight and age group. For each decennial age group, the columns represent the mean arterial pressure of men in the weight groups $<130,130-149,150-169$. $170-189$ and 190 and over, reading from left to right (see Table VII).

TABLE V

RELATION BETWEEN SYSTOLIC AND DIASTOLIC PRFSSURE READINGS OF TWELVE OBSERVERS

\begin{tabular}{|c|c|c|c|c|c|c|c|c|c|}
\hline \multirow{3}{*}{ Observer } & \multicolumn{4}{|c|}{ Males aged 20-39 } & \multirow{3}{*}{ Observer } & \multicolumn{4}{|c|}{ Males aged 40-59 } \\
\hline & \multicolumn{2}{|c|}{ Systolic Pressure } & \multicolumn{2}{|c|}{ Diastolic Pressure } & & \multicolumn{2}{|c|}{ Systolic Pressure } & \multicolumn{2}{|c|}{ Diastolic Pressure } \\
\hline & $\mathrm{mm} . \mathbf{H g}$ & Rank Order & $\mathrm{mm} . \mathbf{H g}$ & Rank Order & & $\mathrm{mm} . \mathbf{H g}$ & Rank Order & $\mathbf{m m} \cdot \mathbf{H g}$ & Rank Order \\
\hline $\begin{array}{l}\mathbf{A} \\
\mathbf{B} \\
\mathbf{C} \\
\mathbf{D} \\
\mathbf{E} \\
\mathbf{F} \\
\mathbf{G} \\
\mathbf{H} \\
\mathbf{I} \\
\mathbf{J} \\
\mathbf{K} \\
\mathbf{L}\end{array}$ & $\begin{array}{l}120 \cdot 0 \\
121 \cdot 7 \\
122 \cdot 4 \\
124 \cdot 8 \\
125 \cdot 2 \\
126 \cdot 8 \\
127 \cdot 8 \\
129 \cdot 5 \\
130 \cdot 0 \\
131 \cdot 3 \\
132 \cdot 2 \\
135 \cdot 4\end{array}$ & $\begin{array}{r}1 \\
2 \\
3 \\
4 \\
5 \\
6 \\
7 \\
8 \\
9 \\
10 \\
11 \\
12\end{array}$ & $\begin{array}{l}72 \cdot 4 \\
69 \cdot 3 \\
68 \cdot 9 \\
74 \cdot 6 \\
79 \cdot 4 \\
71 \cdot 4 \\
68 \cdot 0 \\
81 \cdot 5 \\
72 \cdot 1 \\
72 \cdot 4 \\
68 \cdot 6 \\
78 \cdot 8\end{array}$ & $\begin{array}{c}7 \cdot 5 \\
4 \\
3 \\
9 \\
11 \\
5 \\
1 \\
12 \\
6 \\
7 \cdot 5 \\
? ? \\
10\end{array}$ & $\begin{array}{l}\mathbf{A} \\
\mathbf{B} \\
\mathbf{D} \\
\mathbf{E} \\
\mathbf{I} \\
\mathbf{C} \\
\mathbf{G} \\
\mathbf{F} \\
\mathbf{J} \\
\mathbf{H} \\
\mathbf{L} \\
\mathbf{K}\end{array}$ & $\begin{array}{l}125 \cdot 2 \\
126 \cdot 8 \\
130 \cdot 3 \\
132 \cdot 6 \\
135 \cdot 0 \\
136 \cdot 6 \\
136.7 \\
136 \cdot 8 \\
137.6 \\
139 \cdot 3 \\
140.2 \\
141 \cdot 2\end{array}$ & $\begin{array}{r}1 \\
2 \\
3 \\
4 \\
5 \\
6 \\
7 \\
8 \\
9 \\
10 \\
11 \\
12\end{array}$ & $\begin{array}{l}78 \cdot 3 \\
74 \cdot 9 \\
78 \cdot 9 \\
84 \cdot 2 \\
75 \cdot 6 \\
76 \cdot 6 \\
75 \cdot 6 \\
77 \cdot 7 \\
76 \cdot 8 \\
90 \cdot 7 \\
83 \cdot 0 \\
76 \cdot 5\end{array}$ & $\begin{array}{l}8 \\
1 \\
9 \\
11 \\
2 \cdot 5 \\
5 \\
2 \cdot 5 \\
7 \\
6 \\
12 \\
10 \\
4\end{array}$ \\
\hline
\end{tabular}


TABLE VI

RELATION BETWEEN ARTERIAL PRESSURE AND WEIGHT IN MALES

\begin{tabular}{|c|c|c|c|c|c|c|c|c|c|}
\hline \multirow{2}{*}{$\begin{array}{l}\text { Age Group } \\
\text { (yrs) }\end{array}$} & \multirow{2}{*}{\multicolumn{3}{|c|}{$\begin{array}{c}\text { Mean Arterial Pressure } \\
\text { (mm. Hg) }\end{array}$}} & \multicolumn{5}{|c|}{ Weight (lb.) } & \multirow{2}{*}{$\begin{array}{c}\text { Correlation Coefficient (r) } \\
\text { Systolic Pressure and } \\
\text { Weight }\end{array}$} \\
\hline & & & & $<130$ & 130 & 150 & 170 & $\begin{array}{l}190 \text { and } \\
\text { Over }\end{array}$ & \\
\hline $20-29$ & $\begin{array}{l}\text { Systolic } \\
\text { Diastolic } \\
\text { Number }\end{array}$ & $\begin{array}{l}\cdots \\
\cdots \\
\cdots\end{array}$ & $\begin{array}{l}\cdots \\
\cdots\end{array}$ & $\begin{array}{c}124 \cdot 3 \\
74 \cdot 6 \\
(114)\end{array}$ & $\begin{array}{c}125 \cdot 2 \\
73 \cdot 3 \\
(388)\end{array}$ & $\begin{array}{c}126 \cdot 1 \\
75 \cdot 7 \\
(421)\end{array}$ & $\begin{array}{l}129 \cdot 2 \\
77 \cdot 3 \\
(227)\end{array}$ & $\begin{array}{r}131 \cdot 9 \\
81 \cdot 4 \\
(110)\end{array}$ & $+\cdot 134$ \\
\hline $30-39$ & $\begin{array}{l}\text { Systolic } \\
\text { Diastolic } \\
\text { Number }\end{array}$ & $\begin{array}{l}\cdots \\
\cdots \\
\cdots\end{array}$ & $\begin{array}{l}\cdots \\
\cdots\end{array}$ & $\begin{array}{l}126 \cdot 0 \\
75 \cdot 4 \\
(94)\end{array}$ & $\begin{array}{r}126 \cdot 8 \\
78 \cdot 2 \\
(277)^{2}\end{array}$ & $\begin{array}{c}128 \cdot 3 \\
79 \cdot 1 \\
(429)\end{array}$ & $\begin{array}{c}130 \cdot 9 \\
81 \cdot 6 \\
(263)\end{array}$ & $\begin{array}{r}129 \cdot 2 \\
82 \cdot 1 \\
(178)\end{array}$ & $+\cdot 079$ \\
\hline $40-49$ & $\begin{array}{l}\text { Systolic } \\
\text { Diastolic } \\
\text { Number }\end{array}$ & $\begin{array}{l}\cdots \\
\cdots \\
\cdots\end{array}$ & $\begin{array}{l}\cdots \\
\cdots \\
\cdots\end{array}$ & $\begin{array}{l}128 \cdot 3 \\
79 \cdot 6 \\
(93)\end{array}$ & $\begin{array}{r}128 \cdot 3 \\
79 \cdot 8 \\
(250)\end{array}$ & $\begin{array}{c}129 \cdot 8 \\
81 \cdot 3 \\
(324)\end{array}$ & $\begin{array}{c}133 \cdot 9 \\
83 \cdot 4 \\
(237)\end{array}$ & $\begin{array}{c}140 \cdot 4 \\
87 \cdot 6 \\
(149)\end{array}$ & $+\cdot 208$ \\
\hline $50-59$ & $\begin{array}{l}\text { Systolic } \\
\text { Diastolic } \\
\text { Number }\end{array}$ & $\begin{array}{l}\cdots \\
\cdots \\
\cdots\end{array}$ & $\begin{array}{l}\cdots \\
\cdots\end{array}$ & $\begin{array}{c}137 \cdot 6 \\
81 \cdot 3 \\
(105)\end{array}$ & $\begin{array}{c}135 \cdot 6 \\
81 \cdot 7 \\
(222)\end{array}$ & $\begin{array}{c}138 \cdot 9 \\
85 \cdot 7 \\
(295)\end{array}$ & $\begin{array}{c}142 \cdot 0 \\
87 \cdot 9 \\
(172)\end{array}$ & $\begin{array}{c}145 \cdot 0 \\
89 \cdot 4 \\
(91)\end{array}$ & $+\cdot 128$ \\
\hline 60-69 & $\begin{array}{l}\text { Systolic } \\
\text { Diastolic } \\
\text { Number }\end{array}$ & $\begin{array}{l}\cdots \\
\cdots \\
\cdots\end{array}$ & $\begin{array}{l}\cdots \\
\cdots\end{array}$ & $\begin{array}{c}146 \cdot 7 \\
86 \cdot 1 \\
(47)\end{array}$ & $\begin{array}{c}146 \cdot 0 \\
86 \cdot 8 \\
(92)\end{array}$ & $\begin{array}{c}147 \cdot 6 \\
88 \cdot 8 \\
(110)\end{array}$ & $\begin{array}{l}151 \cdot 7 \\
89 \cdot 8 \\
(63)\end{array}$ & $\bar{C}^{*}$ & $+\cdot 087$ \\
\hline
\end{tabular}

* In Tables VI, VII, and VIII, means are not given for less than forty observations.

As in the Bergen survey (Bøe and others, 1957) and the Report of the Society of Actuaries (1959), both systolic and diastolic readings increased with increasing weight.

However, the increase, although fairly consistent, was not very great-the correlation $(r)$ between systolic pressure and weight ranged from $+\cdot 079$ to $+\cdot 208$ for the five age groups. A similar pattern was uncovered for the relation between pressure reading and arm circumference (Table VII). For each age group mean systolic and diastolic pressure increased fairly regularly with increasing arm size, but again the magnitude of the increase was not impressive and the correlation coefficients ranged only from +.028 to $+\cdot 191$. The relation between height and blood pressure was even less impressive, but showed an interesting age pattern: for the three age groups below 50 correlation coefficients were positive, and for the two older age groups they were negative (Table VIII, overleaf).

These associations cannot be accepted at their face value, for the variables concerned are themselves inter-related. Table IX (overleaf) gives zero order correlations between weight and height, weight and arm circumference, and arm circumference and height. As would be expected (and as is well known) weight and height are closely related. For the five decennial age groups of the present survey, the correlation coefficients ranged from $+\cdot 386$ to $+\cdot 508$, with no evidence of an age trend. Perhaps

TABLE VII

RELATION BETWEEN ARTERIAL PRESSURE AND ARM CIRCUMFERENCE

\begin{tabular}{|c|c|c|c|c|c|c|c|c|c|}
\hline \multirow{2}{*}{$\underset{\text { (yrs) }}{\text { Age Group }}$} & \multirow{2}{*}{\multicolumn{2}{|c|}{$\begin{array}{c}\text { Mean Arterial } \\
\text { Pressure } \\
\text { (mm. Hg) }\end{array}$}} & \multicolumn{6}{|c|}{ Arm Circumference (cm.) } & \multirow{2}{*}{$\begin{array}{l}\text { Correlation Coefficient ( } r) \\
\text { Systolic Pressure and } \\
\text { Arm Circumference }\end{array}$} \\
\hline & & & $<24$ & 24 & $26-$ & 28- & 30 & $\begin{array}{l}32 \text { and } \\
\text { Over }\end{array}$ & \\
\hline $20-29$ & $\begin{array}{l}\text { Systolic } \\
\text { Diastolic } \\
\text { Number }\end{array}$ & $\begin{array}{l}\cdots \\
\cdots\end{array}$ & $\begin{array}{l}124 \cdot 1 \\
75 \cdot 2 \\
(46)\end{array}$ & $\begin{array}{l}125.0 \\
73.9 \\
(252)\end{array}$ & $\begin{array}{l}125 \cdot 3 \\
74 \cdot 6 \\
(430)\end{array}$ & $\begin{array}{l}127 \cdot 6 \\
75 \cdot 6 \\
(310)\end{array}$ & $\begin{array}{l}130 \cdot 6 \\
79 \cdot 2 \\
(170)\end{array}$ & $\begin{array}{l}131 \cdot 3 \\
81: 7 \\
(51)\end{array}$ & $+\cdot 125$ \\
\hline $30-39$ & $\begin{array}{l}\text { Systolic } \\
\text { Diastolic } \\
\text { Number }\end{array}$ & $\begin{array}{l}. \\
\therefore \\
\therefore\end{array}$ & $\overline{(23)}$ & $\begin{array}{l}126 \cdot 8 \\
77 \cdot 5 \\
(112)\end{array}$ & $\begin{array}{l}127 \cdot 1 \\
78 \cdot 1 \\
(361)\end{array}$ & $\begin{array}{l}129 \cdot 2 \\
79 \cdot 6 \\
(394)\end{array}$ & $\begin{array}{l}130 \cdot 5 \\
82 \cdot 2 \\
(255)\end{array}$ & $\begin{array}{l}127 \cdot 9 \\
80 \cdot 3 \\
(98)\end{array}$ & $+\cdot 057$ \\
\hline $40-49$ & $\begin{array}{l}\text { Systolic } \\
\text { Diastolic } \\
\text { Number }\end{array}$ & $\begin{array}{l}\ldots \\
\cdots \\
\cdots\end{array}$ & $\overline{-}$ & $\begin{array}{l}127 \cdot 3 \\
79 \cdot 2 \\
(106)\end{array}$ & $\begin{array}{l}129 \cdot 6 \\
80 \cdot 4 \\
(279)\end{array}$ & $\begin{array}{l}131 \cdot 6 \\
82 \cdot 5 \\
(348)\end{array}$ & $\begin{array}{l}134 \cdot 8 \\
83 \cdot 9 \\
(206)\end{array}$ & $\begin{array}{l}140 \cdot 1 \\
87 \cdot 5 \\
(88)\end{array}$ & $+\cdot 191$ \\
\hline $50-59$ & $\begin{array}{l}\text { Systolic } \\
\text { Diastolic } \\
\text { Number }\end{array}$ & $\begin{array}{l}\cdots \\
\therefore\end{array}$ & $\overline{(37)}$ & $\begin{array}{l}138 \cdot 2 \\
81 \cdot 5 \\
(113)\end{array}$ & $\begin{array}{l}136 \cdot 2 \\
82 \cdot 6 \\
(278)\end{array}$ & $\begin{array}{l}139 \cdot 0 \\
86 \cdot 5 \\
(283)\end{array}$ & $\begin{array}{l}143 \cdot 3 \\
88 \cdot 6 \\
(131)\end{array}$ & $\begin{array}{l}149 \cdot 8 \\
91 \cdot 7 \\
(42)\end{array}$ & $+\cdot 118$ \\
\hline $60-69$ & $\begin{array}{l}\text { Systolic } \\
\text { Diastolic } \\
\text { Number }\end{array}$ & $\begin{array}{l}\ldots \\
\cdots\end{array}$ & $\overline{\bar{z}}$ & $\begin{array}{l}147 \cdot 0 \\
86 \cdot 7 \\
(60)\end{array}$ & $\begin{array}{l}147 \cdot 4 \\
87.5 \\
(106)\end{array}$ & $\begin{array}{l}149 \cdot 0 \\
89 \cdot 4 \\
(101)\end{array}$ & $\overline{(3 \overline{6})}$ & $\frac{\overline{7}}{(8)}$ & $+\cdot 028$ \\
\hline
\end{tabular}


TABLE VIII

RELATION BETWEEN ARTERIAL PRESSURE AND HEIGHT

\begin{tabular}{|c|c|c|c|c|c|c|c|c|c|}
\hline \multirow{2}{*}{$\begin{array}{c}\text { Age Group } \\
(\mathrm{yrs})\end{array}$} & \multirow{2}{*}{\multicolumn{2}{|c|}{$\begin{array}{l}\text { Mean Arterial } \\
\text { Pressure } \\
\text { (mm. Hg) }\end{array}$}} & \multicolumn{6}{|c|}{ Hẹght (in.) } & \multirow{2}{*}{$\begin{array}{c}\text { Correlation Coefficient ( } r) \\
\text { Systolic Pressure and } \\
\text { Height }\end{array}$} \\
\hline & & & $<64$ & 64 & $66-$ & $68-$ & $70-$ & $\begin{array}{l}72 \text { and } \\
\text { Over }\end{array}$ & \\
\hline $20-29$ & $\begin{array}{l}\text { Systolic } \\
\text { Diastolic } \\
\text { Number }\end{array}$ & $\begin{array}{l}\cdots \\
\cdots \\
\cdots\end{array}$ & $\overline{(39)}$ & $\begin{array}{r}124 \cdot 9 \\
75 \cdot 1 \\
(142)\end{array}$ & $\begin{array}{c}127 \cdot 2 \\
75 \cdot 7 \\
(301)\end{array}$ & $\begin{array}{r}127 \cdot 7 \\
75 \cdot 7 \\
(374)\end{array}$ & $\begin{array}{r}125 \cdot 8 \\
75 \cdot 7 \\
(251)\end{array}$ & $\begin{array}{r}127 \cdot 3 \\
76 \cdot 2 \\
(153)\end{array}$ & +.024 \\
\hline $30-39$ & $\begin{array}{l}\text { Systolic } \\
\text { Diastolic } \\
\text { Number }\end{array}$ & $\begin{array}{l}\cdots \\
\cdots\end{array}$ & $\begin{array}{c}127 \cdot 1 \\
78 \cdot 3 \\
(63)\end{array}$ & $\begin{array}{r}127 \cdot 0 \\
78 \cdot 7 \\
(179)\end{array}$ & $\begin{array}{r}128 \cdot 5 \\
79 \cdot 6 \\
(368)\end{array}$ & $\begin{array}{c}128 \cdot 7 \\
79 \cdot 6 \\
(351)\end{array}$ & $\begin{array}{l}129 \cdot 5 \\
80 \cdot 0 \\
(200)\end{array}$ & $\begin{array}{l}130 \cdot 2 \\
80 \cdot 7 \\
(82)\end{array}$ & +.055 \\
\hline $40-49$ & $\begin{array}{l}\text { Systolic } \\
\text { Diastolic } \\
\text { Number }\end{array}$ & $\begin{array}{l}\cdots \\
\cdots \\
\cdots\end{array}$ & $\begin{array}{l}132 \cdot 4 \\
83 \cdot 8 \\
(90)\end{array}$ & $\begin{array}{c}132 \cdot 2 \\
82 \cdot 2 \\
(205)\end{array}$ & $\begin{array}{r}130 \cdot 5 \\
82 \cdot 3 \\
(304)\end{array}$ & $\begin{array}{c}131 \cdot 2 \\
81 \cdot 9 \\
(267)\end{array}$ & $\begin{array}{c}131 \cdot 6 \\
80 \cdot 8 \\
(144)\end{array}$ & $\begin{array}{c}140 \cdot 8 \\
85 \cdot 0 \\
(43)\end{array}$ & +.029 \\
\hline $50-59$ & $\begin{array}{l}\text { Systolic } \\
\text { Diastolic } \\
\text { Number }\end{array}$ & $\begin{array}{l}\cdots \\
\cdots\end{array}$ & $\begin{array}{c}142 \cdot 1 \\
84 \cdot 8 \\
(126)\end{array}$ & $\begin{array}{c}139 \cdot 4 \\
84 \cdot 9 \\
(215)\end{array}$ & $\begin{array}{c}137 \cdot 8 \\
85 \cdot 4 \\
(255)\end{array}$ & $\begin{array}{c}139 \cdot 7 \\
84 \cdot 8 \\
(175)\end{array}$ & $\begin{array}{c}136 \cdot 2 \\
85 \cdot 3 \\
(78)\end{array}$ & $\overline{(37)}$ & -.032 \\
\hline $60-69$ & $\begin{array}{l}\text { Systolic } \\
\text { Diastolic } \\
\text { Number }\end{array}$ & $\begin{array}{l}\ldots \\
\cdots \\
\cdots\end{array}$ & $\begin{array}{c}149 \cdot 1 \\
90 \cdot 1 \\
(83)\end{array}$ & $\begin{array}{c}152 \cdot 4 \\
88 \cdot 8 \\
(103)\end{array}$ & $\begin{array}{c}144 \cdot 9 \\
85 \cdot 5 \\
(84)\end{array}$ & $\begin{array}{c}144 \cdot 8 \\
87 \cdot 9 \\
(48)\end{array}$ & $\overline{-}$ & $\overline{-}$ & -.097 \\
\hline
\end{tabular}

TABLE IX

ZERO ORDER CORRELATION COEFFICIENTS FOR HEIGHT, WEIGHT, AND ARM CIRCUMFERENCE

\begin{tabular}{|c|c|c|c|}
\hline \multirow{2}{*}{$\begin{array}{c}\text { Age } \\
\text { Group } \\
\text { (yrs) }\end{array}$} & \multicolumn{3}{|c|}{ Correlation Coefficients } \\
\hline & $\begin{array}{c}\text { Weight/ } \\
\text { Arm Circumference }\end{array}$ & Weight/Height & 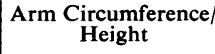 \\
\hline $\begin{array}{l}20-29 \\
30-39 \\
40-49 \\
50-59 \\
60-69\end{array}$ & $\begin{array}{l}\cdot 735 \\
\cdot 738 \\
\cdot 769 \\
\cdot 762 \\
\cdot 750\end{array}$ & $\begin{array}{l}.432 \\
.508 \\
.483 \\
.499 \\
.386\end{array}$ & $\begin{array}{l}\cdot 098 \\
\cdot 199 \\
\cdot 236 \\
\cdot 236 \\
\cdot 187\end{array}$ \\
\hline
\end{tabular}

less expected and certainly less well known is the fact that weight and arm circumference are even more closely associated ( $r$ ranged from +.735 to $+\cdot 769$ ). The relation between arm circumference and height, although also positive, was less striking.

In Table $X$ an attempt has been made to separate the effect of these associations by the method of partial correlation. When the effects of height and arm circumference were held constant, the association between systolic pressure and weight remained significantly positive only for men aged 40 years and over. Under 40 years of age, the effect of weight upon pressure was negligible. When weight and height were held constant, the association between pressure and arm circumference almost disappeared. It would seem, therefore, that in the present survey the greater part of the positive association observed between arm circumference and sphygmomanometer reading was indirect and attributable to the correlation of arm circumference with weight. The relation between pressure and height is interesting, but difficult to interpret. When weight and arm circumference were held constant, blood pressure tended to decrease with increasing height, especially among middle-aged and elderly men.

\section{INFLUENCE OF OCCUPATION}

In this section, the relation between the men's arterial pressure and the physical and mental demands of their occupations is explored. The

TABLE X

CRUDE (ZERO) AND CORRECTED (SECOND ORDER) CORRELATION COEFFICIENTS FOR SYSTOLIC PRESSURE AND WEIGHT, ARM CIRCUMFERENCE, AND HEIGHT

\begin{tabular}{|c|c|c|c|c|c|c|c|}
\hline \multirow{3}{*}{ Age Group (yrs) } & \multicolumn{7}{|c|}{ Correlation Coefficient } \\
\hline & \multicolumn{2}{|c|}{ Systolic Pressure/Weight } & \multicolumn{2}{|c|}{$\begin{array}{l}\text { Systolic Pressure/Arm } \\
\text { Circumference }\end{array}$} & \multicolumn{2}{|c|}{ Systolic Pressure/Height } & \multirow{2}{*}{ Standard Error } \\
\hline & Crude & Corrected & Crude & Corrected & Crude & Corrected & \\
\hline $\begin{array}{l}20-29 \\
30-39 \\
40-49 \\
50-59 \\
60-69\end{array}$ & $\begin{array}{l}\cdot 134^{*} \\
\cdot 079^{*} \\
\cdot 208^{*} \\
\cdot 128^{*} \\
\cdot 087\end{array}$ & $\begin{array}{l}.067 \\
.035 \\
.122^{*} \\
.106^{*} \\
\cdot 151^{*}\end{array}$ & $\begin{array}{l}\cdot 125^{*} \\
.057^{*} \\
.191^{*} \\
.118^{*} \\
.028\end{array}$ & $\begin{array}{r}.027 \\
.004 \\
.029 \\
.002 \\
-.082\end{array}$ & $\begin{array}{c}.024 \\
.055 \\
.029 \\
-.032 \\
-.097\end{array}$ & $\begin{array}{r}-.026 \\
.018 \\
-.075 \\
-.108^{*} \\
-\cdot 154^{*}\end{array}$ & $\begin{array}{l} \pm .028 \\
\pm .028 \\
\pm .031 \\
\pm .034 \\
\pm .055\end{array}$ \\
\hline
\end{tabular}


physical demands made upon each man by his work were classified as sedentary, light, or moderate to severe, and the mental demands as minimum, moderate, or severe, by the person immediately superior to him in the industrial hierarchy. For example, a foreman made the assessment for each man in his section, superintendents assessed the work of foremen, and factory managers the work of superintendents. As Brown and others (1957) have pointed out in respect of a similar classification, "the possible criticism of such criteria is not that they are inaccurate but that they are crude" and consequently "it is perhaps permissible to have more confidence in positive than in negative findings".

Table XI and Fig. 3 show that, below age 40, there was no obvious relation between arterial pressure and the physical demands of occupations but, for each of the three decades above 40 , men in sedentary occupations had on the average the lowest pressures and men in moderate to heavy occupations the highest pressures, while men doing light work occupied an intermediate position. Since sedentary occupations tend to be more intellectually exacting than occupations which are physically demanding, it is not surprising that the blood pressure of men over 40 was inversely related to the mental demands their work made upon them (Table XII). Amongst middle-aged and elderly men the difference between the mean pressure of those doing sedentary and those doing moderate to heavy work, and between those whose jobs were intellectually exacting and those who work made minimum mental demands, was no more than a few millimetres, but the direction of the trend is in agreement with that demonstrated

TABLE XI

\begin{tabular}{|c|c|c|c|}
\hline \multirow{2}{*}{$\begin{array}{l}\text { Mean Arterial } \\
\text { Pressure } \\
(\mathrm{mm} . \mathbf{H g})\end{array}$} & \multicolumn{3}{|c|}{ Physical Demands of Occupation } \\
\hline & Sedentary & Light & $\begin{array}{c}\text { Moderate to } \\
\text { Heavy }\end{array}$ \\
\hline $\begin{array}{ll}\text { Systolic } & \ldots \\
\text { Diastolic } & \ldots \\
\text { Number } & \ldots\end{array}$ & $\begin{array}{l}127 \cdot 1 \\
76 \cdot 5 \\
(373)\end{array}$ & $\begin{array}{l}125 \cdot 9 \\
76 \cdot 3 \\
(436)\end{array}$ & $\begin{array}{l}127 \cdot 2 \\
74 \cdot 2 \\
(451)\end{array}$ \\
\hline $\begin{array}{l}\text { Systolic } \\
\text { Diastolic }\end{array}$ & $\begin{array}{l}129 \cdot 4 \\
80 \cdot 9 \\
(244)\end{array}$ & $\begin{array}{l}128 \cdot 3 \\
79 \cdot 2 \\
(384)\end{array}$ & $\begin{array}{l}128 \cdot 3 \\
79 \cdot 3 \\
(616)\end{array}$ \\
\hline $\begin{array}{ll}\text { Systolic } & \ldots \\
\text { Diastolic } & \ldots \\
\text { Number } & \ldots\end{array}$ & $\begin{array}{l}129 \cdot 5 \\
81.6 \\
(193)\end{array}$ & $\begin{array}{l}131 \cdot 2 \\
82 \cdot 2 \\
(319)\end{array}$ & $\begin{array}{l}132.9 \\
82.4 \\
(544)\end{array}$ \\
\hline $\begin{array}{l}\text { Systolic } \\
\text { Diastolic } \\
\text { Number }\end{array}$ & $\begin{array}{c}136 \cdot 8 \\
84 \cdot 1 \\
(200)\end{array}$ & $\begin{array}{l}139 \cdot 7 \\
85 \cdot 4 \\
(274)\end{array}$ & $\begin{array}{l}140 \cdot 3 \\
85 \cdot 3 \\
(413)\end{array}$ \\
\hline $\begin{array}{ll}\text { Systolic } & \ldots \\
\text { Diastolic } & \ldots \\
\text { Number } & \ldots\end{array}$ & $\begin{array}{l}143 \cdot 5 \\
85 \cdot 4 \\
(79)\end{array}$ & $\begin{array}{l}148 \cdot 7 \\
88 \cdot 5 \\
(102)\end{array}$ & $\begin{array}{c}151 \cdot 6 \\
88 \cdot 9 \\
(152)\end{array}$ \\
\hline
\end{tabular}

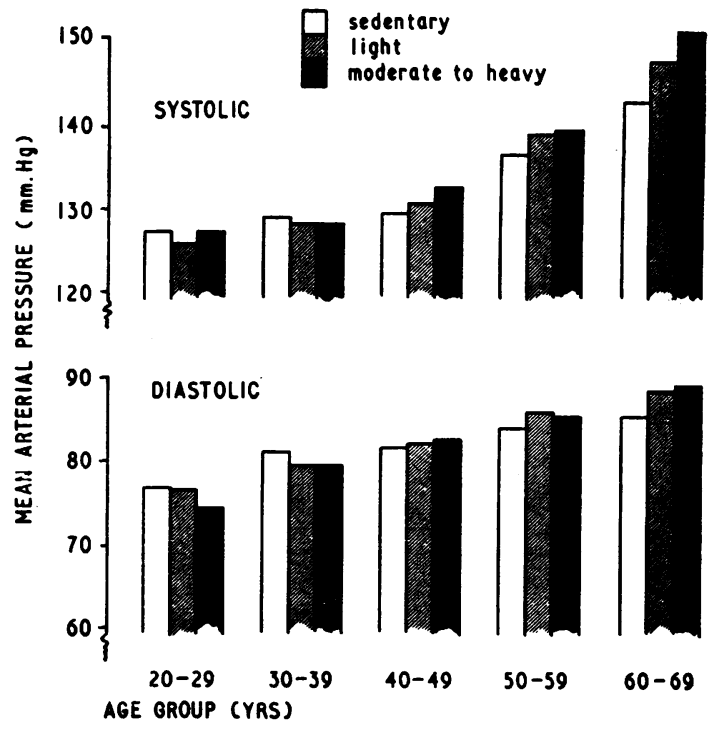

FIG. 3.-Mean arterial pressure related to physical demands of occupation and age group.

TABLE XII

RELATION BETWEEN ARTERIAL PRESSURE AND MENTAL DEMANDS OF OCCUPATION

\begin{tabular}{|c|c|c|c|c|}
\hline \multirow{2}{*}{$\begin{array}{c}\text { Age } \\
\text { Group } \\
\text { (yrs) }\end{array}$} & \multirow{2}{*}{$\begin{array}{c}\text { Mean Arterial } \\
\text { Pressure } \\
(\mathrm{mm} . \mathbf{H g})\end{array}$} & \multicolumn{3}{|c|}{ Mental Demands of Occupation } \\
\hline & & Minimum & Moderate & Severe \\
\hline $20-29$ & $\begin{array}{ll}\text { Systolic } & . \\
\text { Diastolic } & \ldots \\
\text { Number } & \ldots\end{array}$ & $\begin{array}{l}127 \cdot 4 \\
75 \cdot 3 \\
(393)\end{array}$ & $\begin{array}{l}126 \cdot 8 \\
76 \cdot 0 \\
(724)\end{array}$ & $\begin{array}{l}124.4 \\
74.8 \\
(143)\end{array}$ \\
\hline $30-39$ & $\begin{array}{ll}\text { Systolic } & \ldots \\
\text { Diastolic } & \ldots \\
\text { Number } & \ldots\end{array}$ & $\begin{array}{l}127.4 \\
79.7 \\
(296)\end{array}$ & $\begin{array}{l}128 \cdot 8 \\
79 \cdot 3 \\
(842)\end{array}$ & $\begin{array}{l}129 \cdot 7 \\
81 \cdot 6 \\
(106)\end{array}$ \\
\hline $40-49$ & $\begin{array}{ll}\text { Systolic } & \ldots \\
\text { Diastolic } & \ldots \\
\text { Number } & .\end{array}$ & $\begin{array}{l}133 \cdot 7 \\
83 \cdot 1 \\
(324)\end{array}$ & $\begin{array}{l}131 \cdot 1 \\
82 \cdot 0 \\
(594)\end{array}$ & $\begin{array}{l}129 \cdot 5 \\
80 \cdot 7 \\
(138)\end{array}$ \\
\hline $50-59$ & $\begin{array}{l}\text { Systolic } \\
\text { Diastolic } \ldots \\
\text { Number }\end{array}$ & $\begin{array}{l}139 \cdot 7 \\
85 \cdot 5 \\
(295)\end{array}$ & $\begin{array}{l}139 \cdot 1 \\
85 \cdot 6 \\
(476)\end{array}$ & $\begin{array}{r}137 \cdot 5 \\
84 \cdot 6 \\
(116)\end{array}$ \\
\hline $60-69$ & $\begin{array}{ll}\text { Systolic } & \ldots \\
\text { Diastolic } & \ldots \\
\text { Number } & \ldots\end{array}$ & $\begin{array}{c}147 \cdot 6 \\
88 \cdot 5 \\
(129)\end{array}$ & $\begin{array}{l}145 \cdot 5 \\
88 \cdot 6 \\
(163)\end{array}$ & $\begin{array}{c}136 \cdot 7 \\
83 \cdot 5 \\
(41)\end{array}$ \\
\hline
\end{tabular}

in Birmingham by Edwards and others (1959) and contrary to that suggested by the data collected in South Wales by Miall and Oldham (1958).

Table XIII (overleaf) shows that men in sedentary occupations were, on the average, a few pounds heavier than men whose jobs made moderate to heavy physical demands, and Table XIV (overleaf) shows that they were also considerably taller. Since weight and height are closely related, it is evident that occupation has to be considered in terms of body build rather than of weight or height by themselves. But to define body build with any precision multiple 
TABLE XIII

RELATION BETWEEN PHYSICAL DEMANDS OF OCCUPATION AND WEIGHT (lb.)

\begin{tabular}{|c|c|c|c|}
\hline \multirow{2}{*}{$\underset{\text { (yrs) }}{\text { Age. Group }}$} & \multicolumn{3}{|c|}{ Physical Demands of Occupation } \\
\hline & Sedentary & Light & $\begin{array}{c}\text { Moderate to } \\
\text { Heavy }\end{array}$ \\
\hline $\begin{array}{l}20-29 \\
30-39 \\
40-49 \\
50-59 \\
60-69\end{array}$ & $\begin{array}{l}159 \cdot 6 \\
164 \cdot 6 \\
162 \cdot 1 \\
160 \cdot 9 \\
155 \cdot 4\end{array}$ & $\begin{array}{l}158 \cdot 1 \\
165 \cdot 1 \\
163 \cdot 5 \\
161 \cdot 1 \\
154 \cdot 2\end{array}$ & $\begin{array}{l}155 \cdot 7 \\
161 \cdot 3 \\
161 \cdot 9 \\
155 \cdot 9 \\
154 \cdot 5\end{array}$ \\
\hline
\end{tabular}

In Tables XIII, XIV, and XV, the number of men in each age and occupational group is the same as for the corresponding group in Table XI.

TABLE XIV

RELATION BETWEEN PHYSICAL DEMANDS OF OCCUPATION AND HEIGHT (in.)

\begin{tabular}{c|c|c|c}
\hline \multirow{2}{*}{$\begin{array}{c}\text { Age Group } \\
\text { (yrs) }\end{array}$} & \multicolumn{2}{|c|}{ Physical } & Demands of Occupation \\
\cline { 2 - 3 } & Sedentary & Light & $\begin{array}{c}\text { Moderate to } \\
\text { Heavy }\end{array}$ \\
\hline $20-29$ & $69 \cdot 41$ & $68 \cdot 95$ & $68 \cdot 14$ \\
$30-39$ & $68 \cdot 85$ & $68 \cdot 18$ & $67 \cdot 76$ \\
$40-49$ & 67.99 & $67 \cdot 74$ & $67 \cdot 22$ \\
$50-59$ & $67 \cdot 64$ & $66 \cdot 99$ & $66 \cdot 40$ \\
$60-69$ & $66 \cdot 60$ & $65 \cdot 54$ & $65 \cdot 30$ \\
& & & \\
\hline
\end{tabular}

measurements are called for and, unfortunately, in the Birmingham survey only weight, height, and arm circumference were taken. However, if a single index of body build has to be chosen, the ponderal index $(\mathrm{Ht} \div \sqrt[3]{ } \mathrm{Wt})$ is perhaps the least objectionable. This has been related to occupation in Table XV, which shows that for each of the five decennial age groups men doing moderate to heavy work had on the average a lower ponderal index than men in sedentary occupations. Since the relation between ponderal index and obesity is an inverse one-thin persons have a high index and obese persons have a low onethis indicates that men in heavy occupations were heavier, height for height, than men in less active occupations. We must therefore now examine the hypothesis that differences in physique are sufficient to explain the observed increase in the arterial pressure of middle-aged and elderly men as the physical demands of their occupations increased.

TABLE XV

RELATION BETWEEN PHYSICAL DEMANDS OF OCCUPATION AND PONDERAL INDEX $\left(\mathrm{Ht} \div^{2} \sqrt{ } \mathrm{Wt}\right)$

\begin{tabular}{c|c|c|c}
\hline \multirow{2}{*}{$\begin{array}{c}\text { Age Group } \\
\text { (yrs) }\end{array}$} & \multicolumn{2}{|c|}{ Physical Demands of Occupation } \\
\cline { 2 - 3 } & Sedentary & Light & $\begin{array}{c}\text { Moderate to } \\
\text { Heavy }\end{array}$ \\
\hline $20-29$ & $12 \cdot 86$ & $12 \cdot 81$ & $12 \cdot 71$ \\
$30-39$ & 12.63 & $12 \cdot 50$ & $12 \cdot 51$ \\
$40-49$ & 12.53 & 12.46 & $12 \cdot 41$ \\
$50-59$ & 12.52 & $12 \cdot 38$ & 12.40 \\
$60-69$ & 12.46 & $12 \cdot 32$ & $12 \cdot 24$ \\
\hline
\end{tabular}

To this end the trivial effect of arm circumference was ignored and, in the first place, the regression of systolic pressure in $\mathrm{mm}$. $\mathrm{Hg}(s)$ on weight in pounds $(w)$ and height in inches $(h)$ was calculated for the age groups 40-49, 50-59, and 60-69:

$$
\begin{aligned}
& s(40-49)=0 \cdot 195 w-0 \cdot 702 h+147 \cdot 43 \\
& s(50-59)=0 \cdot 183 w-1 \cdot 112 h+184 \cdot 65 \\
& s(60-69)=0 \cdot 169 w-1 \cdot 702 h+234 \cdot 54
\end{aligned}
$$

Expected values for the systolic pressure of men in sedentary, light, and moderate to heavy occupations in the two age groups were then obtained by substituting in these equations observed mean weights and heights from Tables XIII and XIV. For example, the mean weight of men aged 40-49 in sedentary occupations was $162 \cdot 1 \mathrm{lb}$. (Table XIII) and their height was 67.99 in. (Table XIV). Substituting these values in the appropriate equation we get:

$$
\begin{aligned}
s & (40-49, \text { sedentary }) \\
& =0 \cdot 195 \times 162 \cdot 1-0 \cdot 702 \times 67 \cdot 99+147 \cdot 43 \\
& =131 \cdot 3 .
\end{aligned}
$$

Similarly, the expected systolic pressure for men aged 40-49 in light occupations was $131 \cdot 8$ and in moderate to heavy occupations also $131 \cdot 8$. This is to say that, because of their different physique, men aged $40-49$ in moderate to heavy occupations might have been expected to have had a mean systolic pressure $0.5 \mathrm{~mm}$. higher $(131 \cdot 8-131 \cdot 3)$ than men in sedentary occupations; it was, in fact, $3.4 \mathrm{~mm}$. higher $(132 \cdot 9-129 \cdot 5)$. In the same way the expected and observed differences were respectively 0.5 and $3.5 \mathrm{~mm}$. for men aged $50-59$, and 2.1 and

\begin{tabular}{|c|c|c|c|c|c|}
\hline \multirow[b]{2}{*}{$\begin{array}{c}\text { Age } \\
\text { Group } \\
\text { (yrs) }\end{array}$} & \multirow[b]{2}{*}{$\begin{array}{c}\text { Systolic } \\
\text { Pressure } \\
(\mathbf{m m} \text {. Hg) }\end{array}$} & \multicolumn{4}{|c|}{ Physical Demands of Occupation } \\
\hline & & $\begin{array}{c}(a) \\
\text { Sedentary }\end{array}$ & $\begin{array}{c}\text { (b) } \\
\text { Light }\end{array}$ & $\begin{array}{c}(c) \\
\text { Moderate } \\
\text { to Heavy }\end{array}$ & $\begin{array}{l}\text { Difference } \\
\text { between } \\
\text { moderate to } \\
\text { heavy and } \\
\text { sedentary } \\
(c-a)\end{array}$ \\
\hline $40-59$ & $\begin{array}{l}\text { Observed } \\
\text { Expected }\end{array}$ & $\begin{array}{l}129 \cdot 5 \\
131 \cdot 3\end{array}$ & $\begin{array}{l}131 \cdot 2 \\
131 \cdot 8\end{array}$ & $\begin{array}{r}132.9 \\
131 \cdot 8\end{array}$ & $\begin{array}{l}3 \cdot 4 \\
0 \cdot 5\end{array}$ \\
\hline $50-59$ & $\begin{array}{l}\text { Observed } \\
\text { Expected }\end{array}$ & $\begin{array}{l}136 \cdot 8 \\
138 \cdot 9\end{array}$ & $\begin{array}{l}139 \cdot 7 \\
139 \cdot 6\end{array}$ & $\begin{array}{l}140 \cdot 3 \\
139 \cdot 3\end{array}$ & $\begin{array}{l}3 \cdot 5 \\
0 \cdot 6\end{array}$ \\
\hline $60-69$ & $\begin{array}{l}\text { Observed } \\
\text { Expected }\end{array}$ & $\begin{array}{l}143 \cdot 5 \\
147 \cdot 4\end{array}$ & $\begin{array}{l}148 \cdot 7 \\
149 \cdot 0\end{array}$ & $\begin{array}{l}151 \cdot 6 \\
149 \cdot 5\end{array}$ & $\begin{array}{l}8 \cdot 1 \\
2 \cdot 1\end{array}$ \\
\hline
\end{tabular}
$8.1 \mathrm{~mm}$. for men aged 60-69 (Table XVI).

TABLE XVI

DIFFERENCE BETWEEN OBSERVED* AND EXPECTED $\dagger$ SYSTOLIC PRESSURE IN RELATION TO PHYSICAL DEMANDS OF OCCUPATION

* See Table XI.

t Obtained by substituting the observed values for $w$ (Table XIII) and $h$ (Table XIV) in the appropriate multiple regression equation. 
Evidently the fact that middle-aged and elderly men in moderate to heavy occupations were heavier, height for height, than men in sedentary occupations does not explain their higher arterial pressures.

\section{Discussion}

The preceding analysis relates to single, casual, arterial pressure measurements taken by twelve medical practitioners on a sample of men at their place of work. The data are imperfect and the survey leaves many questions unanswered. Nevertheless, the analysis has thrown some light on the interrelation between blood pressure, age, physique, and occupation.

The survey has clarified a little the confusion about the relative importance of weight and arm circumference in their influence upon sphygmomanometer readings. Weight and arm circumference are highly correlated (Table XI) and, when the influence of weight upon systolic pressure is held constant, the influence of arm circumference practically disappears. It seems therefore that the greater part of the observed relation between arm girth and sphygmomanometer reading is attributable to the close association between arm girth and weight.

The survey has also confirmed what the Bergen survey of Bøe and others (1957) suggested-that height and blood pressure tend to be inversely related, especially among middle-aged and elderly men.

No support has been provided for the widely-held belief that anxiety and mental strain are of importance in the aetiology of hypertension. In fact, the blood pressure of men over 40 was inversely related to the mental demands, and directly related to the physical demands, of their occupations. The trend was not very marked but, whatever the explanation, it was not attributable to the relation between physique and occupation. This finding contradicts the results of the survey of arterial pressure in an urban and a rural population in South Wales reported by Miall and Oldham (1958). The number of men involved in the South Wales survey was very small (89 in light occupations and 152 in heavy occupations), the age range very wide (15-80 years), and the analysis based on the method of age-adjusted scores used by Hamilton and others (1954b). In this method the deviation of each person's pressure from the mean value for his or her age group is multiplied by a factor calculated to give the deviation from the mean which that person would expect to show at some standard age. The use of such an age-adjusted deviation score can perhaps be justified in the situation for which it was devised-the need to compare the pressures of parents and children-but its use by Miall and Oldham (1958) to compare pressure levels in different occupational groups is more difficult to accept. The method is strictly justified only if as he grows older the average man's pressure does in fact follow the regression curve relating pressure with age and, as Miall and Oldham admitted, "it seems certain that this does not happen, particularly as high pressures at any age are apparently associated with a curtailed life expectation".

Perhaps the most striking result of the analysis has been the demonstration of a wide range of observer variation in the recording of arterial pressure by means of stethoscope and mercury sphygmomanometer. Some observers read consistently high and others consistently low, and those who read high for systolic pressure by no means always read high for diastolic pressure. In the present survey the mean systolic pressure of all men aged 20-39 and 40-59 was respectively $127.8 \mathrm{~mm}$. and $135.6 \mathrm{~mm}$., a difference of $7.8 \mathrm{~mm}$., but observers $A$ and $L$ (Table III) respectively recorded a mean pressure of 120.0 and 135.4 (a difference of $15.4 \mathrm{~mm}$.) for their subsamples of men aged $20-39$ and a mean pressure of $125 \cdot 2$ and $140 \cdot 2$ (a difference of $15.0 \mathrm{~mm}$.) for men aged $40-59$.

The magnitude and consistency of this observer variation is of more than theoretical interest, since acceptance for life insurance and employment sometimes turns upon one doctor's interpretation of a sphygmomanometer reading.

\section{SUMmaRY}

(1) Some of the results of a survey of the arterial pressure of 5,239 men and 1,572 women measured at their place of work by twelve medical practitioners are reported.

(2) There were substantial differences between the twelve observers in their auscultatory sphygmomanometer readings of the systolic and diastolic pressures of employees in the same age group, some reading consistently high and others consistently low; the level at which each observer read systolic pressure appeared to be unrelated to the level at which he read diastolic pressure.

(3) Recorded pressures tended to increase with weight and with arm circumference at all ages.

(4) At constant height and weight, however, the relation between pressure and arm circumference was negligible. This suggests that the greater part of the observed increase of sphygmomanometer reading with increasing arm girth is attributable to the close association between arm girth and weight. 
(5) At constant weight and arm circumference, pressure tended to decrease with increasing height, especially for middle-aged and elderly men.

(6) Below the age of 40 , there was no clear relation between arterial pressure and occupation but, for the three decades $40-49,50-59$, and $60-69$, pressure was directly related to physical demands of occupation (being highest among men doing moderate to heavy work) and inversely related to mental demands of occupation (being highest among men whose occupations were not intellectually exacting).

(7) The relation between pressure and occupation was not attributable to the fact that men in heavy occupations tended to be heavier, height for height, than men in sedentary occupations.

It is with pleasure that I acknowledge my indebtedness to the General Electric Company, Limited, and to Dr L. A. McDowell, Consultant Regional Adviser in Mass Radiography, for permission to carry out this survey, and to my colleagues, Dr E. Lewis-Faning, $\operatorname{Dr} \mathrm{H}$.
Campbell, and Mr T. Khosla for their help with, and advice about, the statistical handling of the data.

\section{REFERENCES}

Bøe, J., Humerfelt, S., and Wedervang, F. (1957). Acta med. scand., Suppl. 321.

Brown, R. G., Davidson, L. A. G., McKeown, T., and Whitfield, A. G. W. (1957). Lancet, 2, 1073.

Edwards, F., McKeown, T., and Whitfield, A. G. W. (1959). Clin. Sci., 18, 289.

Hamilton, M., Pickering, G. W., Roberts, J. A. Fraser, and Sowry, G. S. C. (1954a). Ibid., 13, 11.

- - , - - (1954b). Ibid., 13, 37.

Lowe, C. R., and McKeown, T. (1962). Lancet, 1, 1086.

Miall, W. E., and Oldham, P. D. (1958). Clin. Sci., 17, 409.

Morris, J. N., Heady, J. A., Raffle, P. A. B., Roberts, C. G., and Parks, J. W. (1953). Lancet, 2, 1053; 1111.

Morris, J. N., and Crawford, M. D. (1958). Brit. med. J., 2, 1485.

Ragan, C., and Bordley, J. (1941). Bull. Johns Hopk. Hosp., 69, 504.

Registrar General (1954). "Decennial Supplement, England and Wales, 1951", Occupational Mortality, Part 1. HMSO, London.

Society of Actuaries. "Build and Blood Pressure", 1959, I, Chicago. 